\title{
Five Questions Answered: A Review of Autologous Hematopoietic Stem Cell Transplantation for the Treatment of Multiple Sclerosis
}

\author{
Harold L. Atkins ${ }^{1}$ (D) - Mark S. Freedman ${ }^{1}$
}

Published online: 18 August 2017

(C) The American Society for Experimental NeuroTherapeutics, Inc. 2017

\begin{abstract}
Multiple sclerosis (MS) is thought to be an autoimmune disease targeting the central nervous system leading to demyelination, and axonal and neuronal damage, resulting in progressive disability. More intensive therapies such as immunodepletion with hematopoietic stem-cell rescue are being used at a time prior to patients becoming irreversibly disabled. Over the last 15 years, there has been a shift away from using autologous hematopoietic stem-cell transplants (aHSCT) to treat patients with progressive MS, towards treating those with active inflammation and relapses. There is an increasing body of evidence that aHSCT improves all measured MS outcomes, including burden of disease on MRI, clinical relapses, accumulation of disability, and quality of life of patients with active MS not controlled with standard therapy. Importantly, the progression-free survival curves of these patients plateau after the first few years demonstrating the impact that aHSCT has in changing the natural history of MS, potentially freeing patients from the relentless accumulation of disability. Concurrently there has been a reduction in procedure-related mortality. The results of randomized trials will likely spur further development of this field.
\end{abstract}

Keywords Multiple sclerosis $\cdot$ Hematopoietic stem cell transplantation · Immunoablation · Chemotherapy ·

Autoimmunity $\cdot$ Immune reconstitution

Electronic supplementary material The online version of this article (doi:10.1007/s13311-017-0564-5) contains supplementary material, which is available to authorized users.

Harold L. Atkins

hatkins@ohri.ca

1 The Ottawa Hospital, 501 Smyth Rd., Mailstop 926, Ottawa, Ontario K1H 8L6, Canada

\section{Introduction}

Multiple sclerosis (MS) is considered an autoimmune disease where, for still unknown reasons, the immune system targets the central nervous system (CNS) leading to demyelination and ultimately to neuronal and axonal loss, which results in progressive disability. Research has led to the development of more than a dozen approved therapies that aim to control this immune-mediated CNS damage. Unfortunately, for some patients, these medications fail to control the damage and neurologists have sought to implement more intensive therapies before patients become irreversibly disabled. This has led to the emergence of immunodepletion with hematopoietic stem cell rescue as a treatment for patients with aggressive MS [1].

The information reported to international registries about patients with MS that have undergone autologous hematopoietic stem-cell transplantation (aHSCT) suggests a trend to increasing annual activity of the procedure, occurrence across more countries and centers, changes in the type of patients considered for the procedure, and changes in the methods used for transplantation. Over the last 3 years, major journals have published peer-reviewed retrospective cohort studies [2, 3], prospective single arm trials [4-6], and a randomized phase II trial [7] of aHSCT. Observations characterizing the changes in the immune system that occur following aHSCT for MS have been summarized previously [8]; this review focuses on the current understanding of the role of aHSCT in patients with MS.

\section{What Is HSCT?}

In the context of MS, aHSCT is the term used to describe a complex procedure that involves the administration of a "conditioning regimen" composed of cytotoxic chemotherapy 
and biologic agents that target and destroy the immune system followed by infusion of hematopoietic stem cells (HSC) to regenerate or reconstitute the immune system and aid recovery from off-target effects of the chemotherapy (e.g., pancytopenia resulting from marrow suppression or ablation). Both components, the conditioning regimen and the HSC infusion, are important to the therapeutic effect of aHSCT and either when applied alone are ineffective or less effective than the combination $[9,10]$. A key goal of the conditioning regimen is to eliminate ongoing inappropriate immune responses in order to prevent further immune-mediated destruction within the CNS. At the current time, the available agents used in conditioning are nonselective, both eliminating dysfunctional autoreactive immune responses and destroying adaptive or protective immune responses. In addition, these agents have effects beyond cytoreduction of the immune system causing, for instance, marrow aplasia, and gastrointestinal toxicity, which become more pronounced with increasing dose intensity.

\section{Are all HSCT the Same?}

Immunity in the CNS plays an important role in the pathogenesis of MS and the selection of agents that cross the bloodbrain barrier (BBB) should be considered in the design of the conditioning regimen. The chemotherapeutic agents used in conditioning regimens cross the BBB to a limited degree. Pharmacokinetics studies show that cyclophosphamide is able to cross the BBB; however, its active metabolites do this poorly. Busulfan can accumulate in the cerebrospinal fluid with a serum:cerebrospinal fluid ratio of 1.3 [11, 12]. This may be responsible for the risk of seizure following high-dose busulfan administration, and antiseizure prophylaxis is warranted. The ability of biologic agents [e.g., antithymocyte globulin (ATG) or alemtuzumab that have been used in conditioning regimens] to cross the $\mathrm{BBB}$ in this situation has not been studied, although antibodies do not typically cross an intact BBB. While radiation is an effective modality for ablating the immune system, the damaged CNS appears to be sensitive to its harmful effects. Used in early studies, total body irradiation has fallen out of practice owing to suboptimal outcomes [13].

While it is difficult to compare directly the intensity of conditioning regimens that use different agents, intensity is best considered as the overall outcome of the biologic effects of the drugs administered. The effectiveness of conditioning regimens at cytoreducing the immune system and their toxicities generally increase in parallel. The depth and length of neutropenia and thrombocytopenia, the frequency and severity of gastrointestinal toxicity, regimen-related mortality, and length of hospital stay are familiar markers of dose intensity. The most commonly used conditioning regimens can be separated into higher and more moderate dose intensities.
Regimens consisting of carmustine, etoposide, cytarabine, melphalan, and ATG (BEAM-ATG), or busulfan, cyclophosphamide, and ATG (BUCTX-ATG) are more intense than cyclophosphamide-ATG; however, even the latter has the potential to cause significant complications without appropriate supportive care. All conditioning regimens should be administered within a medical unit specialized in the care of patients receiving high-dose therapy and aHSCT.

Most frequently, the HSC graft is autologous, collected from the patient undergoing aHSCT prior to the administration of the conditioning regimen. While direct aspiration from the bone marrow has been used, currently HSC are mobilized from the marrow cavity into the circulation using a recombinant cytokine (e.g., filgrastim or granulocyte colonystimulating factor) or moderate doses of a chemotherapy that is not toxic to HSC, such as cyclophosphamide, in combination with the recombinant cytokine. Filgrastim, when used alone, can cause MS to flare so steroids must be given concurrently $[14,15]$. This leads one to be cautious about using newer mobilizing agents, such as plerixafor - a CXCR4 ana$\log$, which has not been tested for the mobilization of HSC for transplants in autoimmune disease, although it has been shown to be effective in other settings [16]. The HSC graft product, collected by apheresis, is rich in neutrophils, lymphocytes, and monocytes with only $1-5 \%$ of the cells representing hematopoietic stem and precursor cells. Sufficient HSC for the subsequent HSCT are usually collected in 1 to 3 apheresis sessions.

The HSC graft can be collected from a compatible donor, but allogeneic HSCT are associated with a greatly increased morbidity and mortality due to graft-versus-host disease. While some have postulated a graft-versus-autoimmune lymphocyte activity mediated by the allogeneic HSC graft [17], this largely remains theoretical with anecdotal case reports highlighting both the success and failure of controlling the underlying autoimmunity in MS [18].

As autoimmune illnesses can be adoptively transferred to a recipient from an afflicted individual that donates a graft [19], it is possible that viable autoreactive and potentially diseasecausing lymphocytes in an autologous HSC graft can lead to a resurgence of the MS following aHSCT, nullifying the benefits obtained from the lympho-depleting conditioning regimen. The number of autoreactive immune cells in the HSC graft can be reduced in vivo by administering cyclophosphamide during the graft mobilization. This leads to partial lymphocyte reduction in the graft product, although in our experience there are, on average, $46 \times 10^{6}$ lymphocytes $/ \mathrm{kg}$ recipient weight remaining in the graft product. Further immune cell depletion can be accomplished using clinical-scale immunomagnetic selection to positively select the CD34 hematopoietic stem and progenitor cells resulting in HSC grafts almost free of contaminating, potentially disease-causing immune cells [5]. However, the role of ex vivo immune cell 
depletion is controversial. One small randomized trial using autologous HSCT to treat patients with rheumatoid arthritis did not show a difference in outcome between arms [20]. Similarly, the outcome of a retrospective analysis of a cohort of patients with progressive MS undergoing aHSCT did not show a difference between patients that received a CD34 selected HSC graft and those that received an unselected graft [21]. However a cohort that received a CD34 selected aHSCT following busulfan, cyclophosphamide, and antithymocyte globulin conditioning did not have any evidence of new CNS inflammatory activity for up to 13 years [5]. CD34 selection adds technical complexity and increases the cost of the transplant procedure; however, in the correct setting it may prevent adoptive reintroduction of CNS reactive lymphocytes leading to better long-term disease control.

Provided the conditioning regimen is sufficiently intense to abrogate all immune cells from the body, the graft is responsible for immune reconstitution. Homeostatic expansion of transferred mature lymphocytes results in an overlap of the T-cell receptor (TCR) repertoire before and after HSCT, whereas de novo lymphopoiesis from transplanted HSC results in a novel TCR repertoire. Tcell clones that recognize and react to MBP are initially depleted by the conditioning regimen but re-appear over time [22]. After immune reconstitution the MBP reactive lymphocytes are skewed to a Th1 phenotype rather than the Th17 present prior to aHSCT, suggesting that they are the result of de novo lymphopoiesis rather than a "carry over" from before aHSCT [23]. Furthermore, the change in CD4 helper cell phenotype corresponds to the change from active inflammatory MS to long-term remission. Following BEAM-ATG conditioning and CD34 selected HSCT, the CD4 TCR repertoire does not contain dominant TCR clones from before treatment consistent with complete immune ablation and reconstitution of this compartment through de novo lymphopoiesis; however, the reconstituted $\mathrm{CD} 8$ compartment contained clones that were dominant prior to HSCT, suggesting that CD8 lymphocytes may be more resistant to the conditioning regimen [24]. Immune reconstitution is generally associated with restoration of a diverse polyclonal TCR repertoire [23, 25]; however, limited TCR diversity was associated with treatment failure suggesting the persistence of autoreactive disease-causing clones in some patients that receive BEAM-ATG [24]. These results provide support for the use of conditioning that is sufficient to result in complete immune ablation. Recurrence of MS may result from inadequate immune depletion or the persistence of autoreactive clones in the HSC graft. Both the conditioning regimen and the use of a T-cell-replete or T-celldepleted graft can affect the nature of immune reconstitution and MS activity following HSCT but systematic comparative studies have not been performed.

\section{Why Are We Still Even Talking About HSCT?}

There are a large number of disease-modifying drugs available for treatment of MS that have been shown, in high-quality randomized but short-term ( 2 years for most) clinical trials, to reduce the frequency of relapses and slow disease progression in patients with active inflammatory relapsing-remitting MS. Not a single study has shown complete control of all the immune-mediated CNS inflammatory response in patients; at best, they control or minimize the disease. These agents suppress maladaptive immune responses but are nonselective and also affect adaptive immune responses. As they become more potent in altering the immune system they convey a greater incidence of secondary autoimmune sequelae and opportunistic infections. Like cancer therapy, where a complete remission is the first step on the road to cure; suppression of acute inflammatory activity is only the first step to halting disease progression. While effective, suppression of relapses is incomplete with hazard ratios for the reduction in annualized relapse rates of about $50 \%$ for the most active agents versus first-line treatments such as interferon- $\beta$. However, despite the demonstrable benefits of these agents, it has been difficult to show a plateau in the accumulation of further disabilities. Indeed, about $10 \%$ to $15 \%$ of patients have demonstrated progression of disabilities after 2 years of treatment and only $32 \%$ to $47 \%$ of patients show no evidence of disease activity (NEDA; defined as no evidence of relapse, MRI activity, or disease progression over a defined period) portending further decline over time even with the most effective agents $[26,27]$.

HSCT results in NEDA for $78 \%$ to $83 \%$ of MS patients at 2 years and $60 \%$ to $68 \%$ of MS patients at 5 years of follow-up, significantly higher than NEDA reported for patients in clinical trials of new MS drugs [28]. While comparing outcome of reported trials can be fraught with bias, patients entered into trials of aHSCT are at least as, if not more, active than those enrolled in drug trials. More importantly, particularly for those patients with MS treated with a higher-intensity conditioning regimen, such as BEAM-ATG or busulfan-cyclophosphamide-ATG, the progression-free survival curves plateau after the first few years, demonstrating the important impact that aHSCT has in changing the natural history of MS and potentially freeing patients from the relentless accumulation of disability $[5,6]$.

Beyond just stopping further disease progression, longterm follow-up of patients with MS undergoing HSCT demonstrates a substantial proportion have stable long-term improvement in disability as measured by their Expanded Disability Scale Score (EDSS) and improvement in their quality of life as measured by the 36 question Short Form Survey (SF-36). More than half of patients had an improvement in 
their EDSS of 1.0 points or more following cyclophosphamide-ATG conditioning and aHSCT, 15 of 25 patients had similar improvement at their latest follow-up after BEAMATG conditioning and aHSCT, whereas about 35\% demonstrated improved EDSS scores following busulfan, cyclophosphamide-ATG conditioning, and aHSCT [4-6]. The mechanism of recovery is uncertain, but could be related to the stemcell infusion or endogenous repair mechanisms brought into play following suppression of active inflammation in the CNS. A better understanding of the mechanism of this important outcome may allow for selection of patients at a time when they have a greater possibility for recovery.

\section{Who Should Receive HSCT and When Should It Be Performed?}

While the initial studies of aHSCT enrolled patients with progressive MS, over time, aHSCT was used to treat a broader range of patients. Consensus expert opinion led to the publication of guidelines in 2012 [29], recommending that ideal patients to be considered for HSCT are those with: 1) malignant MS, including Marburg-variant MS that presents with rapidly progressive inflammation and demyelination, and develop severe disability within a year; 2) highly active relapsing MS with frequent relapses and developing focal inflammatory lesions on magnetic resonance imaging (MRI) despite treatment with one or more lines of conventional treatment; or 3) progressive MS with ongoing and sustained increase in their disability load only if there are concurrent relapses or focal inflammatory lesions on MRI.

A recently published retrospective analysis by the European Society for Blood and Marrow Transplant Registry and the Center for International Blood and Marrow Research using data from patients that underwent aHSCT for MS confirmed, in a multivariate analysis, that progressionfree survival is better in younger patients, relapsing versus progressive patients, and patients treated with only 1 or 2 lines of therapy prior to proceeding to HSCT [3].

\section{Can HSCT Be Made Less Risky?}

Evidence points to the benefits of aHSCT for MS, yet its application remains limited. Several barriers exist to its broad application, including limitations in the resources of transplant units capable of caring for these patients, unfamiliarity of stem-cell transplant physicians in the assessment and care of patients with MS, unfamiliarity of neurologists with the stemcell transplant procedure, reticence by some healthcare payers to fund the procedure, and concerns about the morbidity and mortality associated with aHSCT. Regimen-related mortality has been reported to be about $4 \%$ for patients undergoing
aHSCT following high-intensity conditioning regimens [5, 30], although there were no regimen-related deaths among the 25 participants in the HALT-MS trial using the same BEAM-based conditioning regimen [6]. Reducing the intensity of the conditioning regimen results in lower mortality with several large cohort studies reporting no deaths attributable to aHSCT $[2,4]$. It remains uncertain if recurrent MS activity differs between patients receiving conditioning regimens with differing intensity. NEDA appears to be similar between patients receiving cyclophosphamide-ATG and BEAM-ATG in an analysis reported by Sormani et al [28]. However, no detectable inflammatory activity was seen in a much longer follow-up of patients receiving an intensive busulfan, cyclophosphamide, and ATG conditioning regimen [5].

Regimen-related morbidity remains another concern as virtually all patients develop grade 3 or 4 nonhematologic toxicities predominantly in the first 30 days after administration of the conditioning regimen [3]. Common acute toxicities include alopecia, neutropenic fevers, sepsis, urinary tract infections, mucositis, and other gastrointestinal toxicities $[5,30]$. Supportive care addressing these issues has improved reducing the burden of symptoms to the point where a few patients at our center have had their entire post-transplant care provided in a day-hospital setting. Late thyroid disease occurs in about $10 \%$ of recipients. Rarely, other autoimmune phenomena, such as immune thrombocytopenic purpura, may occur [4]. Infertility is common in both males and females, and patients should be offered the opportunity for gamete preservation prior to starting chemotherapy treatment. Ovarian failure leading to premature menopause is common. Counselling that includes a thorough explanation of the short- and longterm toxicities and their impact is an important component of care.

Further high-quality information defining the role of aHSCT in the treatment of MS will come from the results of the MIST trial (NCT00273364), a multicenter randomized trial comparing aHSCT following cyclophosphamide-ATG conditioning to treatment with a standard disease-modifying drug. A second multicenter randomized trial comparing a HSCT following BEAM-ATG conditioning to best available drug therapy is currently being developed (Racke, personal communication).

\section{Conclusion}

There is an increasing body of evidence that aHSCT improves the outcome of patients with active MS not controlled with standard therapy. Over the last 15 years, there has been a shift from using aHSCT to treat patients with progressive MS towards treating those with active inflammation and relapses. Concurrently, there has been a reduction in mortality and an improvement in all measured MS 
outcomes, including burden of disease on MRI, clinical relapses, accumulation of disability, and quality of life. The understanding of the mechanisms of immune repair that accompany aHSCT is maturing and could lead to prognostic tests or refinement in the transplant procedure. Just as there is uncertainty about which of the highly active disease-modifying drugs result in the best risk to benefit ratio, it is unclear what the optimal transplant conditioning regimen is. The authors debate between themselves whether HSCT should be reserved for patients with aggressive forms of MS in a phase of the illness where inflammatory activity predominates [1], or whether there is benefit in a risk-adapted approach to selecting a transplant conditioning regimen, using a lower-intensity conditioning regimen for patients with inflammatory but less aggressive relapsing MS, whereas higher-intensity conditioning regimens associated with greater morbidity are reserved for those patients with a more aggressive course. Similarly, if the morbidity of HSCT regimens can be further reduced without compromising its long-term benefits, could autologous HSCT be applied earlier in the treatment paradigm for select patients? The results of randomized trials will spur further development of this field.

Required Author Forms Disclosure forms provided by the authors are available with the online version of this article.

\section{References}

1. Rush CA, MacLean HJ, Freedman MS. Aggressive multiple sclerosis: proposed definition and treatment algorithm. Nat Rev Neurol 2015;11(7):379-389.

2. Burman J, Iacobaeus E, Svenningsson A, et al. Autologous haematopoietic stem cell transplantation for aggressive multiple sclerosis: the Swedish experience. J Neurol Neurosurg Psychiatry 2014;85(10):1116-1121.

3. Muraro PA, Pasquini M, Atkins HL, et al. Long-term outcomes after autologous hematopoietic stem cell transplantation for multiple sclerosis. JAMA Neurol 2017;74(4):459-469.

4. Burt RK, Balabanov R, Han X, et al. Association of nonmyeloablative hematopoietic stem cell transplantation with neurological disability in patients with relapsing-remitting multiple sclerosis. JAMA 2015;313(3):275-284.

5. Atkins HL, Bowman M, Allan D, et al. Immunoablation and autologous haemopoietic stem-cell transplantation for aggressive multiple sclerosis: a multicentre single-group phase 2 trial. Lancet 2016;388(10044):576-585.

6. Nash RA, Hutton GJ, Racke MK, et al. High-dose immunosuppressive therapy and autologous HCT for relapsing-remitting MS. Neurology 2017;88(9):842-852.

7. Mancardi GL, Sormani MP, Gualandi F, et al. Autologous hematopoietic stem cell transplantation in multiple sclerosis: a phase II trial. Neurology 2015;84(10):981-988.

8. Muraro PA, Martin R, Mancardi GL, Nicholas R, Sormani MP, Saccardi R. Autologous haematopoietic stem cell transplantation for treatment of multiple sclerosis. Nat Rev Neurol 2017;13(7): 391-405.
9. Harrison DM, Gladstone DE, Hammond E, et al. Treatment of relapsing-remitting multiple sclerosis with high-dose cyclophosphamide induction followed by glatiramer acetate maintenance. Mult Scler 2012;18(2):202-209.

10. Rice CM, Mallam EA, Whone AL, et al. Safety and feasibility of autologous bone marrow cellular therapy in relapsing-progressive multiple sclerosis. Clin Pharmacol Ther 2010;87(6):679-685.

11. Egorin MJ, Kaplan RS, Salcman M, et al. Cyclophosphamide plasma and cerebrospinal fluid kinetics with and without dimethyl sulfoxide. Clin Pharmacol Ther 1982;32(1):122-128.

12. Hassan M, Oberg G, Ehrsson H, et al. Pharmacokinetic and metabolic studies of high-dose busulphan in adults. Eur J Clin Pharmacol 1989;36(5):525-530.

13. Atkins H. Hematopoietic stem cell transplants (HSCT) for multiple sclerosis (MS) in north America. Bone Marrow Transplant 2010;45:S4-S5.

14. Nash R, Bowen J, McSweeney PA, et al. High-dose immunosuppressive therapy and autologous peripheral blood stem cell transplantation for severe multiple sclerosis. Blood 2003;102(7):23642372.

15. Openshaw H, Stuve O, Antel JP, et al. Multiple sclerosis flares associated with recombinant granulocyte colony-stimulating factor. Neurology 2000;54(11):2147-2150.

16. DiPersio JF, Stadtmauer EA, Nademanee A, et al. Plerixafor and GCSF versus placebo and G-CSF to mobilize hematopoietic stem cells for autologous stem cell transplantation in patients with multiple myeloma. Blood 2009;113(23):5720-5726.

17. Slavin S, Nagler A, Varadi G, Or R. Graft vs autoimmunity following allogeneic non-myeloablative blood stem cell transplantation in a patient with chronic myelogenous leukemia and severe systemic psoriasis and psoriatic polyarthritis. Exp Hematol 2000;28(7):853857.

18. Lu J-Q, Joseph JT, Nash RA, et al. Neuroinflammation and demyelination in multiple sclerosis after allogeneic hematopoietic stem cell transplantation. Arch Neurol 2010;67(6):716-722.

19. Berisso GA, Van Lint MT, Bacigalupo A, Marmont AM. Case report: adoptive autoimmune hyperthyroidism following allogeneic stem cell transplantation from an HLA-identical sibling with Graves' disease. Bone Marrow Transplant 1999;23(10):10911092.

20. Moore J, Brooks P, Milliken S, et al. A pilot randomized trial comparing CD34-selected versus unmanipulated hemopoietic stem cell transplantation for severe, refractory rheumatoid arthritis. Arthritis Rheum 2002;46(9):2301-2309.

21. Fassas A, Anagnostopoulos A, Kazis A, et al. Autologous stem cell transplantation in progressive multiple sclerosis - an interim analysis of efficacy. J Clin Immunol 2000;20(1):24-30.

22. Sun W, Popat U, Hutton G, et al. Characteristics of T-cell receptor repertoire and myelin-reactive $\mathrm{T}$ cells reconstituted from autologous haematopoietic stem-cell grafts in multiple sclerosis. Brain 2004;127(Pt 5):996-1008.

23. Darlington PJ, Touil T, Doucet J-S, et al. Diminished Th17 (not Th1) responses underlie multiple sclerosis disease abrogation after hematopoietic stem cell transplantation. Ann Neurol 2013;73(3): 341-354.

24. Muraro PA, Robins $\mathrm{H}$, Malhotra S, et al. T cell repertoire following autologous stem cell transplantation for multiple sclerosis. J Clin Invest 2014;124(3):1168-1172.

25. Muraro PA, Douek DC, Packer A, et al. Thymic output generates a new and diverse TCR repertoire after autologous stem cell transplantation in multiple sclerosis patients. J Exp Med 2005;201(5): 805-816.

26. Hauser SL, Bar-Or A, Comi G, et al. Ocrelizumab versus interferon beta-1a in relapsing multiple sclerosis. N Engl J Med 2017;376(3): 221-234. 
27. Coles AJ, Twyman CL, Arnold DL, et al. Alemtuzumab for patients with relapsing multiple sclerosis after disease-modifying therapy: a randomised controlled phase 3 trial. Lancet 2012;380(9856):1829 1839.

28. Sormani MP, Muraro PA, Saccardi R, Mancardi G. NEDA status in highly active MS can be more easily obtained with autologous hematopoietic stem cell transplantation than other drugs. Mult Scler 2017;23(2):201-204.
29. Snowden JA, Saccardi R, Allez M, et al. Haematopoietic SCT in severe autoimmune diseases: updated guidelines of the European Group for Blood and Marrow Transplantation. Bone Marrow Transplant 2012;47(6):770-790.

30. Mancardi GL, Sormani MP, Di Gioia M, et al. Autologous haematopoietic stem cell transplantation with an intermediate intensity conditioning regimen in multiple sclerosis: the Italian multicentre experience. Mult Scler 2012;18(6):835-842. 